said, but many times they are unnecessary. From experience during the iast year he is of the opinion that the intranasal mothod he has recommended, where a probe can be passed into the frontal sinus is as safe as any, and the results are gool in the majority of cases. Dr. Ingals has seen only one 'ase in which the results were not good, and that was where some one meddled afterward with the external operation; that calued all the trouble. The suppuration continued and kept discharging through the nose and the patient disappeared with the discharge going on. In that case it was clear that there was some condition that had not been thoroughly attended to at the time of the external operation and perhaps another operation would have been required to clear up the work. Dr. Ingals would not urge that the internal operation is a panacea, but he believes that when the cavity an be entered with the probe it is a safe and good operation and will. cure a large percentage of the cases in a few months, which is as much as can be claimed for the external operation, and there is not the scar.

Dr. Otro T. Frefs, Chicago, said that one point in the Killian operation which he would like to have considered is the removal of the orbit, which seems to him an objectionable feature.

Dr. F. L. JACK, Boston, in closing the discussion, said that he did not lrope to have a discussion on the frontal sinus at all, he simply wanted to explain his cases, which he had hoped to show. There was no question as to the operation. The patients had Iarge orbits and exophthalmos. Some of the cases had lasted for a number of years. He said that his hospital house officer tells him that the cases are perfectly dry, there is no discharge from the nose; he has seen them recently. So far as Dr. Jack has seen the cases there is absolutely no depression. He has one or two eases in which cne would have to get within a foot of the patient in order to sce any scar. Of course there would be no question of a Killian opcration in the class of eases mentioned by Dr. Eagleton. It is the only one to do.

\section{A NEW METHOD OF ANASTOMOSIS OF THE VAS DEFERENS.*}

G. FRANK LYDSTON, M.D.

Professor of Genito-Urinary Surgery and Syphilology, State University of Illinois; Attending Surgcon, St. Mary's and Samaritan Hospitals.

CHICAGo.

With the subsidence of the furore which attended the introduction to the profession of operations on the spermatic cord for prostatic disease, vasectomy was practically relegated to the dead lumber room. While recognizing the fallacy of the claims first made for testicle and cord operations in the relief of prostatic hypertrophy, and having early put myself on record in opposition to the extravagant claims made for such operations, I do not believe that the profession will be inclined to accuse me of over enthusiasm in my endeavor to establish the operation of vasectomy as a legitimate means of relief for certain conditions.

Despite the over enthusiasm which attended the introduction of cord operations, it was not fair to push them aside simply because they did not fulfill the extravagant expectations of those who for one reason or another first advocated them. Whatever may have been the merits of the operation as originally devised, this much must be conceded. riz., that cord operations in suitable eases have a profound influence on the innervation and circulatory supply of the prostate and seminal resicles. Incidentally, a marked effect on nutrition must neessarily result. These offects, which we will term, for

* Read at the Meeting of the lllinois State Vedical Society, May 16. 1906 . the sake of argument, phrsiologic, are not so manifest in elderly patients, in whom the importance of the sixual function is not great, as in younger patients, in whom the sexual function is at its height of activity.

This is an important point, for the value of the cord operations has thus far been estimated from their effects in advanced prostatic diseases in old subjects. These operations have not been employed to any extent in younger patients, in whom beneficial results can be more Jogically expected.

In setting forth the claims of vasectomy as an operation which has a legitimate field in genitourinary work, I wish it to be distinctly understood that $I$ do not adrocate the operation in any case in which there is a reasonable probability of relief being afforded by less radical means. The operation, when properly performed, is practically free from danger. It does not vroduce, as was formerly. supposed, atrophy of the testicle-a condition which, when it follows vasectomy, can be explained on grounds other than disturbed circulation and innervation incidental to the operation on the ras. It is hardly fair, when the operation is clumsily performed, the testicle traumatized, and the tissues perhaps infected, to attribute the resulting atrophy of the testes to the vasectomy.

The principal objection to the operation thus far advanced is its alleged effect on the sexual power. It does not, so far as my experience gocs, produce impotency, and while the bilateral operation necessarily prevents the testicular secretion from taking its normal course, sterility can not fairly be charged up to the operation. if the patient has been impotent prior to its performance. In other words, in most of the cases in which I advocate the performance of vasectomy, the patient is already impotent, and therefore dynamically sterile, and the operation does not add to his woes in these respects.

The most important point of all is the fact that while the operation does not produce permanent sterility, it is by no means necessary that the patient should remain sterile after the operation has accomplished its purpose. It is practicable in probably by far the larger proportion of cases to restore the continuity of one or both vasa deferentia at any time the surgeon may so elect, thus restoring fertility.

The operation of vasectomy has a certain range of application in sexual neurasthenies, spermatophobiacs, and in genuine spermatorrhea. The type of such cases is the individual who has been "quacked" for many years, is impotent, and profoundly neurasthenic, with or without frequent seminal losses. We are wont to say that suggestion and kindly counsel are all that are necessary in the management of such patients. Every practitioner of experience well knows that these means are often ineffective. Treatment, both local and general, is a failure in a certain proportion of such cases.

In a large proportion of cases of pseudo-spermatorrhea, the morale of the patient is so clisturbed by prolonged treatment by quacks and worry that the condition with which we have to deal is one of psychopathia. These patients are impotent and perhaps have even lost sexual desire. More or less frequently recurring nocturnal emissions constitute a symptom which, pooh-pooh it as much as we like, has a very disastrous effect on the mind of the patient. Treat such patients as conscientiously as we may, a certain definite proportion of them fail to find relief. Indecd, no relicf can be afforded the mental condition until the emissions have been checked for a more or less prolonged period. It is 
easy to talk about the efficacy of suggestive therapentics in such cases, but in many of them it is a practical impossibility to accomplish anything so long as the emissions continue; and continue they will, despite all the ordinary methods of relief. Marriage as a specific is not always effective and is often inexcusable. I have seen a number of cases in which it not only was ineffectual, but appeared to aggravate the condition. It is not unusual for individuals who have been long married to become the victims of nocturnal losses as a result of neurasthenia or of some disturbance of the brain or cord due to irritation of the sexual apparatus from one cause or another.

Prostatic inflammation and seminal vesiculitis are perhaps the two most important conditions which give rise to nocturnal emissions. Prostatorrhea, dependent on hyperemia or inflammation of the prostrate, whether or not associated with symptoms of inflammation, is very often exceedingly resistant to treatment. Such patients are usually spermatophobiacs, it is true, but no amount of reasoning suffices to argue them out of the belief that the prostatorrhea constitutes a serious drain on the nervous system. The psychic demoralization of the patient is in many. of these cases very profound. It has been my experience that while the majority of cases of prostatorrhea yield readily to treatment, a certain proportion of cases are absolutely resistant to it. Hypochondriasis, or even typic melancholia, may result, in the foregoing disturbances of the sexual system.

The records of our asylums show only too plainly that definite psychopathia is not infrequently associated with morbid conditions of the sexual, apparatus. I will not take up here the question of the relation of masturbation to cerebral disorders, save to say that, whether it be admitted as a cause of psychopathia or not, it enters very largely into the field of brain abnormality, as it comes under the observation of the alienist.

I believe that, in the cases described, the operation of rasectomy has a perfectly legitimate field. That the result, if beneficial at all, is necessarily due to the psychic impression of the operation is not tenable, in riew of what we know of the physiologic effects of resection of the vasa deferentia. The hyperesthesia of the sexual organs, particularly of the prostatic urethra and seminal vesicles, that exists in these cases, is unquestionably lessened by the operation.

True spermatorrhea is a rare condition, and as intractable as it is rare. That the disease is associated in the majority of cases with cerebrospinal disease of various kinds, is well understood. The fact that the primary disease is of an incurable nature, renders the duty of checking the pathologic seminal discharge none the less imperative in some cases because of oth the physical and psychopathic results which are produced by it. Vasectomy affords in these cases the only hope of relief of what is a very prominent symptom, in the mind of the patient, at least. That the seminal loss is productive of debility, goes without saying.

Since the operation of prostatectomy has been generally adopted by the profession, patients appeal to us for relief of prostatic disease at a much earlier period than formerly. We are often consulted by patients at or but little past middle life, in whom the sexual function is still in full vigor. In many of these cases palliative treatment by massage, sounds, instillation of silver and proper hygiene will give relief and prevent serious trouble developing later on. In some cases, however, despite all our efforts, the prostatic symptoms con- tinue and examination shows progressive enlargement of the prostrate. In such cases we are really not justified in suggesting prostatectomy until other means have been tried. Here, it seems to me, is a most useful field for vasectomy. Although the operation in old prostatic patients has not been followed by the degree of success at first claimed for it, it is far different in relatively young individuals in whom prostatic fibrosis is not yet developed. Here vasectomy offers a more than fair prospect of relief.

In intractable chronic prostatitis and seminal vesiculitis, vasectomy is of the greatest value. Its beneficial effects are due not only to the change which it induers in the circulation and innervation of the prostate, but also to the rest which it secures to the seminal vesicles. Chronic seminal vesiculitis is in some instances absolutely resistant to treatment. The patient is worn out by frequent micturition due to irritation of the vesical neck, with more or less constant pain in that vicinity, radiating into the perineum, testes, penis. Hemorrhagic emissions and functional disturbances of the sexual apparatus are very frequent. Frequent and exhausting nocturnal emissions and painful ejaculation are not rare in such cascs. Patients afflicted in this way are generally

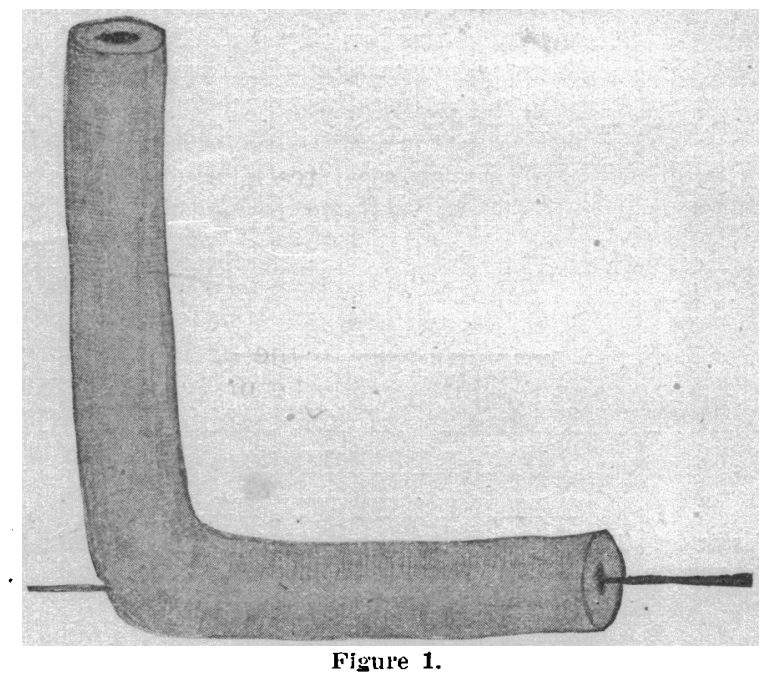

ready to submit to almost anything which offers a prospect of recovery. This has led some over enthusiastic adherents of vesiculectomy and vesiculotomy to propose operation directly on the seminal vesicles. 'This operation is dangerous, difficult, and extremely bloody, and in my opinion should not be resorted to until vasectomy has been given a fair trial. It should be remembered in this connection that the full benefits of vasectomy, even in favorable cases, can not be expected for several months.

In passing, I will call attention to a very important point relative to operations directly on the seminal vesicles, viz., the victims of seminal vesiculitis are often so profoundly neurasthenic that no surgical procedure is likely to give relief. It is by no means consoling to have the annoying symptoms continue after so formidable an operation as vesiculectomy. This is a further argument in favor of a trial of vasectomy before the major operation is resorted to.

A very important field of usefulness for the operation of vasectomy is recurrent epididymitis. In infective conditions of the prostate the epididymis on one or the other side often becomes infected and develops a greater or less degree of inflammation from time to time. In most instances the infective condition of the deep 
urethra on which the epididymitis depends can be corrected. In some cases, however, despite methodic, scientific treatment, the epididymis becomes inflamed at intervals and disables the patient for a greater or less period. This not only occurs in young and middle-aged subjects as a consequence of a specific infection, but in prostatics from pus or septic infection. The prevention of epididymitis in the latter class of cases is, of course, prostatectomy, but there are instances in which the patient is either too young for the operation or refuses to have it performed. Under such circumstances the operation of vasectomy may be performed, with the double object in view of attaining such permanent benefit as may accrue in the particular case so far as the enlargement of the prostate is concerned and the prevention of further attacks of epididymitis. That the integrity of the testes in these cases is in no wise menaced by the operation numerous cases in my own experience testify.

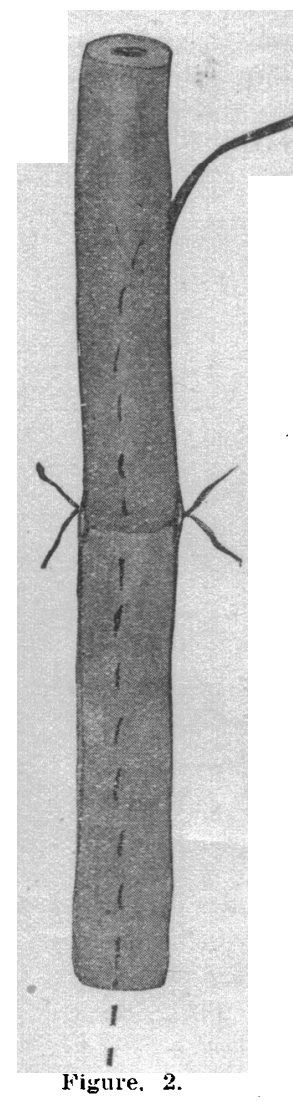

The operation of vasectomy has a certain range of application in suspected or known tuberculosis or malignant lisease of the testes. Cases occur in which the diagnosis is considerably in loubt, although a hard, thickened and perhaps nodular epididymis exists. Under these circumstances the fertility of the affected testis is already nil, so there is nothing to be lost and much to be gained by vasectomy. The operation protects the patient during the period of delay in coming to a positive conclusion as to the nature of the case by shutting off the principal avenue of infection of the genitourinary tract. When a patient with known tuberculosis or malignant tumor of the testis ieclines or defers a radical operation, the operation of vasectomy should be suggested.

I have seen several cases of enlargement of the prostate in which the patient was so debilitated by frequent and severe hemorrhages from the vesical neck that a radical operation was not to be thought of. In these cases I have succeeded by vasectomy in himinishing the frequency and severity of the hemorrhages or checking them altogrther, thus enabling the patient to gain sufficient strength to endure prostatectomy. I recall one case in which the patient had suffered from such severe hemorhages from an extremely large, hard prosiate that I suspected malignant disease. Vasectomy was performed in this case, and about two months later prostatectomy was done. The result of the vasectomy was all that could be desired, and, while the patient was in by no means first-class condition at the time of the prostatectomy, he was sufficiently recuperated from his anemia to render operation justifiable. In cases of enlargement of the prostate where the patient can not or will not have prostatectomy performed, or in which the general condition is so bad that the radical operation does not seem advisabie, vasectomy is inclicated. It is often palliative, and, while I believe the reports of cures accomplished by the operation were undeniably exag- gerated, I believe that the frequency with which benefit is derived from vasectomy warrants the operation under the circumstances outlined.

In inoperable malignant disease of the prostate with severe hemorrhages, vasectomy has, in my exnerience, been beneficial. There is no possible objection tiv the operation, and, therefore, no reason why the natient should not have the benefit of an attempt at palliation by its performance.

\section{TECIINIC OF THE OPERATION.}

After the usual preparation of the scrotum the vas is isolated from the cord and picked up in a fold of skin between the thumb and index finger. As smalt an amount of the skin as possible should be grasped with the vas. A straight needle of good size is now made to transfix the scrotum beneath the vas in such a way that the latter structure remains pinned firmly into the fold of skin and underlying fascia in which it was included by the grasp of the thumb and finger. The vas can now by no possibility slip away from the operator so long as the needle is in situ. An incision from a quarter to a half an inch in length, the smaller the better, is now made parallel with the vas and directly over it. The vas is exposed, separated from the fascia which envelops it, and lifted out of the small wound in the scrotum. It is now stripped for the desired distance until a sufficient extent of it is freed. If it be considered advisable to resect the entire vas up to the inguinal ring, a relatively free incision should be made just below the latter structure, so that the vas may be separated from the cord well up within the inguinal canal. The high incision is only necessary in cases of known or suspected tuberculosis or malignant disease. For ordi-

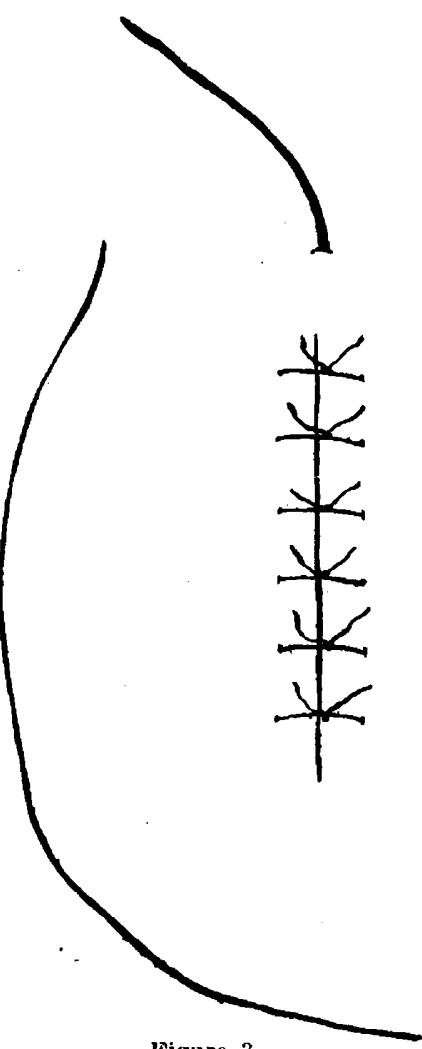

rigure 3. nary purposes, as much of the vas as is necessary can be readily removed through the small incision which I have described. The vas, having been ligated at the points of election by medium-sized catgut, is resected, the lumen of the tubes at the severed ends touched lightly with carbolic acid, and the ends tied together by two of the free ends of catgut used in the ligation. The fascia should be stitched over the ras with fine catgut and the skin wound united with horsehair.

In a certain as yet undetermined proportion of cases it is possible so to anastomose the divided vasa deferentia as to re-establish their continuity. This operation is not necessary save in cases of bilateral accidenta? injury or bilateral vasectomy. It is, however, an operation that should be performed whenever in the course of an operation the vas, even on one side, has been severed. A single permeable vas is sufficient in so far as fertility is 
concerned. Hence it follows that in cases of double resection, followed at a greater or less period by double anastomosis, there are two chances of restoring fertility. One or the other vas is liable to regain patency as the result of anastomosis. The time which should be allowed to elapse following the vasectomy before anastomosis is performed will necessarily vary, according to the amount and character of the improvement in the condition for which the vasectomy was originally performed.

A longitudinal incision from one and a half to two inches in length is made down to the spermatic cord and the vas deferens exposed, freed from its enveloping fascia and lifted out of the wound. The incision is made so that its center corresponds as nearly as possible to the slight nodule on the vas, which indicates the site of the original resection. The vas having been exposed, the nodule is excised. A fine, sharp-pointed probe or long straight needle is now passed into the vas, which is bent at a sufficient angle to facilitate passing the needle through the wall of the duct about an inch and a quarter from its cut extremity (Fig. 1). A strand of the heaviest silkworm gut is now passed into the vas and out of the opening made. by the needle: This can be done with no difficulty whatever. The opposite end of the silkworm gut is made to enter the lumen of the distal end of the vas. The object of this silkworm gut is to preserve the continuity of the lumen of the vas during the process of healing. The vas is now stitehed, end to end, over the coupling of the silkworm gut with very thin catgut (Fig. 2). A portion of the free edge of the fascia which was divided in order to expose the vas deferens is now wrapped about the vas for a short distance above and below the line of anastomosis. The envelopment of fascia is now stitched about the vas deferens in such a way that the line of junction of the vas is completely closed in. The free end of the silkworm gut which has been made to traverse the wall of the vas is now passed from within outward through a puncture in the fascia and skin and made to emerge a short distance above the upper angle of the incision (Fig. 3). Care should be taken not to pull on this strand of silkworm gut, the intention being to allow it to remain within the lumen of the vas until complete union has occurred. The silkworm gut should be removed at the end of a week. The fascia is now stitched with fine catgut and the edges of the scrotal wound brought together with fine silkworm gut or horsehair. The process is repeated upon the opposite vas. There is very little reaction following the operation. although a certain amount of exudation and nodulation about the line of union is perceptible for some weeks after the operation.

Of the six cases which I have operated on in this manner, one has shown microscopically a restoration of the function of the vas on one or the other or both sides by the presence of spermatozoids in the semen. Of the other five, one is apparently a failure, and I have had no opportunity to determine the condition of two. The last two cases were too recent for consideration here.

Unrecognized Causes of Cough.-G. S. Ingersoll, in Mass. Medical Journal, June, 1906, deacribes two unrecognized or unusual causes of cough: 1, Hypertrophied glossal papillæ, overlapping an epiglottis which is bent far forward but otherwise normal; 2, a congenitally asymmetrical epiglottis, which has been made still longer by inflammation, caused by constant friction with the tongue.

\section{ELBOW-JOINT INJURIES, WITH REPORT OF CASES.}

STEWART L. MCCURDY, M.D.

Surgeon P., C., C. \& St. L. Ry.; Surgeon to Presbyterian and Columbia Hospitals: Professor Orthopedic and Clinical Surgery. West. Pennsylvania Medical College.

pittsiurg, PA.

Elbow-joint injuries are troublesome and frequently leave the arm in a most useless condition. Causes for such a condition are fracture into the joint, with displacement of fragments, dislocation forward of the head of the radius, and pathologic ankylosis. In all of these conditions when it is impossible to use the hand while dressing and eating, any operation that will place the hand back into service will be acceptable to unfortunates, and recommended by physicians.

CASE 1.-A young man received the contents of a shotgun in the elbow, the upper part of the radius and ulna being torn off. Figure 1 shows that these bones were thrown above the condyles of the humerus. The operation consisted in squaring all the bones so that their ends could rest against each other. This required the removal of the condyles of the humerus at about the diaphyso-epiphyseal line, and the removal of the upper end of the bones of the forearm. The wound healed promptly. Figure 2 shows that extension is complete. Flexion

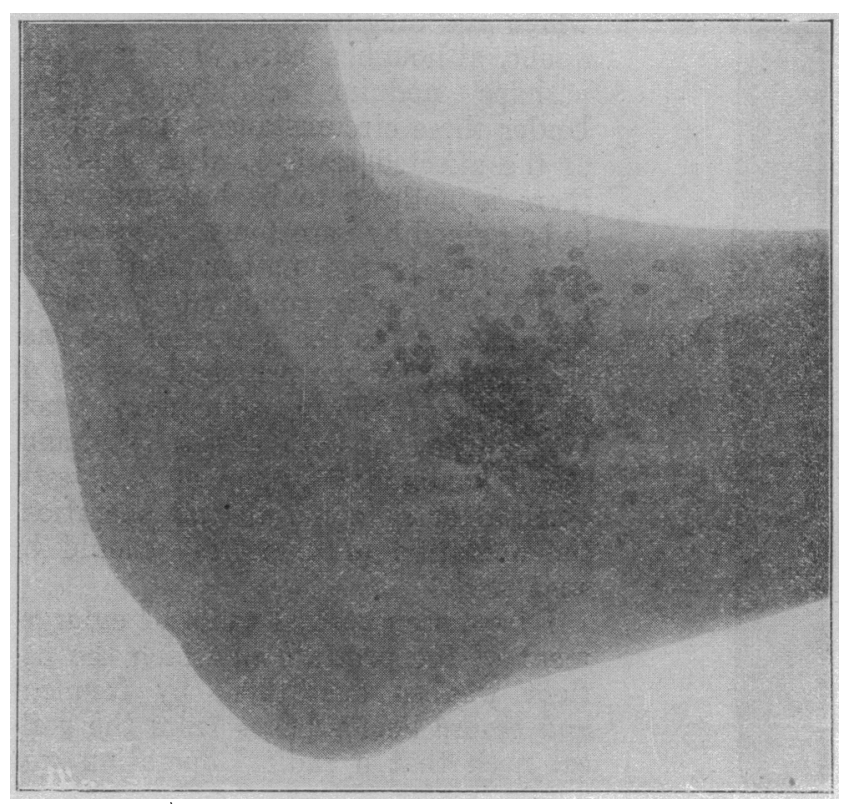

Figure 1.

is somewhat better than shown in Figure 1. The usefulness of the forearm is greatly increased.

CASE 2.-Another case of equal interest is that of a young man who received a compound dislocation of the elbow joint and fracture of the olecranon process. The external condyle, including the entire radial articulation, was broken away. This, as you can see, means that the origin of the entire extensor group of muscles from the external condyle was destroyed, all of these muscles being lacerated extensively. The forearm was turned inward to a right angle with the arm.

The patient expected amputation when he came to the hospital. The operation that was done, however, was first to wire the fractured olecranon (Fig. 3). All of the muscles that were so extensively lacerated were brought back into position and sutured to the soft structures that remained about the external condyle, some to the periosteum of the supracondylolual ridge, others to what remained of the orbicular and ex. ternal lateral ligament. The forearm was placed in extreme extension, as all elbow joint fractures should be placed. The forearm in semipronation so that the head of the radius might rest as near as possible in its normal position, or, in this case, 